\title{
ORIGINAL ARTICLE Identification of temporal genes involved in the mechanisms of spinal cord injury
}

\author{
S Ma, J Wang, L Liu, L Xia and R Tao
}

Objectives: As a high-cost neurological disability, spinal cord injury (SCI) can result in permanent paralysis and loss of sensation. To identify the temporal genes involved in the pathogenesis of SCl, we analysed the expression profile of GSE45006.

Methods: GSE45006 was downloaded from Gene Expression Omnibus, including $20 \mathrm{SCl}$ samples (samples at 1 day, 3 days, 1 week, 2 weeks and 8 weeks after injury, four repetitions for each time point) and 4 normal samples. The Bayesian Estimation of Temporal Regulation (BETR) and randomForest packages were used to screen the temporal genes and the top 100 temporal genes, respectively. Then, the gplots package and Pearson correlation analysis separately were used to perform hot map analysis and expression pattern clustering for the top 100 temporal genes. Using the clusterProfiler package and TargetMine tool, their potential functions were analysed by enrichment analyses. Moreover, interaction relationships between these temporal genes and pathways were investigated by pathway-gene crosslinking networks.

Results: In total, 1907 temporal genes were identified. The top 100 temporal genes were obtained and divided into six clusters. Most of the gene functions were enriched in biological process categories. ARG1 and NOS 3 in cluster 4 were enriched in biological process of arginine catabolic process. TGF $\beta 2$, TGF $33, A L D H 2$ and $A L D H 3 A 2$ were correlated with numerous pathways in the pathway-gene crosslinking network. Pathways related to TGF $\beta 2$ and TGF 33 were connected to pathways related to ARG1 and NOS3 via ARG1. Conclusion: Several temporal genes, including TGF 2 2, TGF 33 , ALDH2, ALDH3A2, ARG1 and NOS3, might be involved in SCI. Spinal Cord (2017) 55, 355-361; doi:10.1038/sc.2016.183; published online 10 January 2017

\section{INTRODUCTION}

Spinal cord injury (SCI) is a type of damage to the nerve tract that runs from the lower back to the brain. ${ }^{1}$ As a high-cost neurological disability, ${ }^{2}$ SCI can even lead to permanent paralysis and loss of sensation. ${ }^{3}$ Evidence has shown that SCI is correlated with lots of genetic factors. It has been reported that interleukin-10 (IL-10) can reduce injury-induced inflammation, thus limiting the extent of neuronal injury and the development of injury-induced pain behaviours following excitotoxic SCI. ${ }^{4,5}$ Schwann cell transplantation combined with glial cell line-derived neurotrophic factor administration may contribute to axonal regeneration and myelin formation following SCI. ${ }^{6}$ Permeable peptide 1 -superoxide dismutase 1 protein can significantly decrease apoptosis of motor neurons induced by reactive oxygen species, which is helpful for functional recovery following SCI. ${ }^{7,8}$ Signal transducer and activator of transcription 3 is an important mediator of reactive astrocytes during the healing process of SCI and may be a promising target for intervention therapy in central nervous system injury. ${ }^{9,10}$ Sry-related HMG box-9 (SOX9) inhibition decreases the levels of chondroitin sulphate proteoglycans and enhances motor function, indicating that SOX9 inhibition is a promising therapeutic strategy for SCI. ${ }^{11}$ However, the genetic mechanisms underlying SCI have not been comprehensively revealed. Thus, it is urgent to investigate the pathogenesis of SCI and develop new therapies.

The need for biologically relevant animal SCI models has focused on the development of animal injury models. Techniques such as weight drop, clip compression and chemically mediated SCI have been successfully introduced into laboratory animal models. ${ }^{12}$ Among these injury models, the clip compression model is introduced as one of the nontransection models of SCI in rodents. ${ }^{12,13}$ The clip compression model has the ability to mimic injury at different levels by adjusting the force and duration of clip application. ${ }^{12,14}$ Using the clip compression model, Chamankhah et al. ${ }^{12}$ perform microarray data analysis, time-series expression profile clustering and functional enrichment analysis to provide an overall picture of biological processes and the relevant molecular events underlying SCI model rats. However, the mechanisms of SCI are not entirely clear. As different analysis procedures and methods can generate different results, we further screened the top 100 temporal genes using the data from Chamankhah et al. ${ }^{12}$ Then, gene clustering of the top 100 temporal genes was performed. The potential functions of these temporal genes were predicated by functional, pathway and protein domain enrichment analyses. In addition, a pathway-gene crosslinking network was constructed to investigate the interaction relationships between these temporal genes and pathways.

\section{MATERIALS AND METHODS}

\section{Microarray data}

The expression profile of GSE45006 deposited by Chamankhah et al. ${ }^{12}$ was downloaded from the Gene Expression Omnibus (GEO, http://www.ncbi.nlm. nih.gov/geo/) database, which was based on the platform of GPL 1355 [Rat230_2] Affymetrix Rat Genome 2302.0 Array. GSE45006 included 
20 SCI samples (including samples at 1 day, 3 days, 1 week, 2 weeks and 8 weeks after injury, four repetitions for each time point) and 4 normal samples. The rats used in this study were female Wistar rats (250 g, Charles River Laboratories (Wilmington, MA, USA), four injured rats for each time point and four sham rats). Injuries were caused by the aneurysm clip method. ${ }^{15,16}$ Briefly, under a $1: 1$ mixture of $\mathrm{O}_{2} / \mathrm{N}_{2} \mathrm{O}$, the rats were drugged with halothane (1-2\%), and the surgical area was disinfected using $70 \%$ ethanol and betadine. An incision was made in the midline of thoracic area (T4-T9), and superficial muscles and skin were retracted. Rats were subject to a T6-T8 laminectomy, and they subsequently received a 35-g clip (Walsh) compression injury at T7 for $1 \mathrm{~min}$. The rats had a cut sutured and were administered Clavamox for 7 days, standard post-operative analgesia treatments and saline $(0.9 \% ; 5 \mathrm{ml})$

\section{Temporal genes screening}

After GSE45006 was downloaded, microarray data were pre-processed according to the following processes. Combined with the annotation information in the platform, probe names were transformed into gene symbols. Using aggregate function methods, ${ }^{17}$ the mean value of probes mapped with the same gene was obtained and taken as the gene expression value. Using the KMN method of the impute package ${ }^{18}$ in $\mathrm{R}$ ( $k$ was set to 10$)$, the probe without a corresponding value was added with difference value. Then, quantile normalisation was conducted by the median method of the preprocessCore package $^{19}$ in $\mathrm{R}$ to obtain a normalised matrix.

The analysis of variance method of the genefilter package ${ }^{20}$ in $\mathrm{R}$ was used to filter genes with a larger interblock difference than intra-group difference. The significance threshold was set to 0.05 . As an improved and sensitive empirical Bayes approach, the Bayesian Estimation of Temporal Regulation (BETR) was utilised to measure differential expression in time-series data. ${ }^{21}$ The BETR algorithm of the BETR package ${ }^{21}$ in $\mathrm{R}$ was applied to calculate probabilities of differentially expressed genes in microarray time-course data. A probability $>0.95$ was used as the cutoff criterion. The classification algorithm random forest is appropriate for gene expression profile data, given that it can be used for a larger number of variables and problems with more than two classes. The algorithm performs well regardless of whether predictive variables are noise, and evaluates variable importance. ${ }^{22}$ Using the randomForest package ${ }^{22}$ in $\mathrm{R}$, the top 100 temporal genes were identified by the random forest method.

\section{Expression pattern clustering of temporal genes}

The gplots package ${ }^{23}$ in $\mathrm{R}$ was used to perform heatmap analysis for the expression patterns of the top 100 temporal genes and to construct two-dimensional hierarchical clustering figures. Subsequently, Pearson correlation coefficient, which is one of the most common coefficients used to evaluate the dependence of two variables, ${ }^{24}$ was employed to cluster the expression patterns of the top 100 temporal genes.

\section{Functional, pathway and protein domain enrichment analysis}

Gene Ontology (GO) is one of the most important tools used to represent and process information concerning genes, gene functions and gene products. ${ }^{25}$ The Kyoto Encyclopedia of Genes and Genomes (KEGG) is a database comprised of known genes and their biochemical functionalities. ${ }^{26}$ Using the clusterProfiler package $^{27}$ in R, GO and KEGG pathway enrichment analyses were performed for the top 100 temporal genes. $P$-values were adjusted using the Benjamini and Hochberg test ${ }^{28}$ and an adjusted $P$-value $<0.05$ was used as the cutoff criterion. In addition, the TargetMine online tool ${ }^{29}$ was used to conduct GO, KEGG pathway and protein domain enrichment analyses for the genes in clusters. $P$-values were adjusted using the Holm-Bonferroni method, ${ }^{30}$ and an adjusted $P$-value $<0.05$ was used as the threshold.

\section{Pathway-gene crosslinking network construction}

Cytoscape is a popular software package that can integrate high-throughput expression data or other molecular networks with biomolecular interaction networks into a unified biological network. ${ }^{31}$ After pathway enrichment analysis was performed for the genes in clusters, Cytoscape software ${ }^{31}$ (http://www. cytoscape.org) was used to visualise the pathway-gene crosslinking network.

\section{RESULTS}

Temporal genes analysis

After analysis using the betr package in R, a total of 1907 temporal genes with a probability $\geqslant 0.95$ were identified from the microarray data of GSE45006. Then, the top 100 temporal genes were obtained using the randomForest package in $\mathrm{R}$.

\section{Expression pattern clustering of temporal genes}

Two-dimensional hierarchical clustering features of expression patterns of the top 100 temporal genes are presented in a heatmap (Figure 1). The hierarchical clustering tree (Figure 2) showed that the top 100 temporal genes were classified into six clusters (cluster 1-6) according to their expressions in samples at each time point. The heatmaps of the six clusters are presented in Figure 3. From 1 day to 8 weeks after injury, the expressions of genes in cluster 1 and cluster 2 decreased firstly and then gradually increased, in contrast to the genes in cluster 6. After the introduction of clip compression injury and up to 8 weeks after injury, the expressions of genes in cluster 3 gradually decreased along with the experimental duration. Gene expression levels in normal samples were significantly reduced compared with those in SCI models at each time point. In cluster 4, gene expression levels in SCI models at 1 day and 3 days post injury were significantly increased compared with those in SCI models at other time points and normal samples. The expression of genes in cluster 5 gradually increased, and gene expression levels in normal samples were significantly increased compared with those in SCI models at each time point.

\section{Functional, pathway and protein domain enrichment analysis}

The top 10 GO terms enriched for the top 100 temporal genes are listed in Table 1. Most of the GO terms were in biological process categories, including localisation $(P$-value $=8.29 \mathrm{E}-08)$, single-organism process $(P$-value $=2.52 \mathrm{E}-07)$ and response to organic substance $(P$-value $=9.93 \mathrm{E}-07)$. The KEGG pathways enriched for the top 100 temporal genes are listed in Table 2, including arginine and proline metabolism $(P$-value $=2.37 \mathrm{E}-03)$, colorectal cancer $(P$-value $=2.37 \mathrm{E}-03)$ and glycerolipid metabolism $(P$-value $=8.63 \mathrm{E}-03)$.

Using the TargetMine online tool, GO functional, KEGG pathway and protein domain enrichment analyses were performed for the genes in the six clusters (Table 3). Two genes in cluster 1 (CD4 and VCAM1) were involved in immunoglobulin $\mathrm{C} 2$-set domain $(P$-value $=1.48 \mathrm{E}-02)$. Three genes in cluster $2(C T S H, R N P E P$ and TPP1) were enriched in molecular function of exopeptidase activity $(P$-value $=4.20 \mathrm{E}-02)$. Ten genes in cluster 3 were involved in cellular component of cytosol $(P$-value $=1.33 \mathrm{E}-02)$. Moreover, arginase 1 (ARG1) and nitric oxide synthase 3 (NOS3) in cluster 4 were enriched in biological processes of arginine catabolic process $(P$-value $=3.77 \mathrm{E}-03)$, arginine metabolic process $(P$-value $=5.65 \mathrm{E}-03)$ and glutamine family amino acid catabolic process $(P$-value $=4.53 \mathrm{E}-02)$. There were no terms enriched for the genes in cluster 5 and cluster 6 .

\section{Construction of a pathway-gene crosslinking network}

The pathway-gene crosslinking network was constructed following pathway enrichment analysis for the top 100 temporal genes in clusters (Figure 4). As a result, transforming growth factor beta 2 (TGF $\beta 2)$; $T G F \beta 3$, aldehyde dehydrogenase $2(A L D H 2)$; and aldehyde dehydrogenase 3 family, member A2 ( $A L D H 3 A 2)$ were involved in numerous pathways. Both TGFB2 and TGFB3 were associated with map05146 amoebiasis pathway. In addition, $A L D H 3 A 2$ and $A L D H 2$ are associated with both map01100 metabolic pathways and map00330 

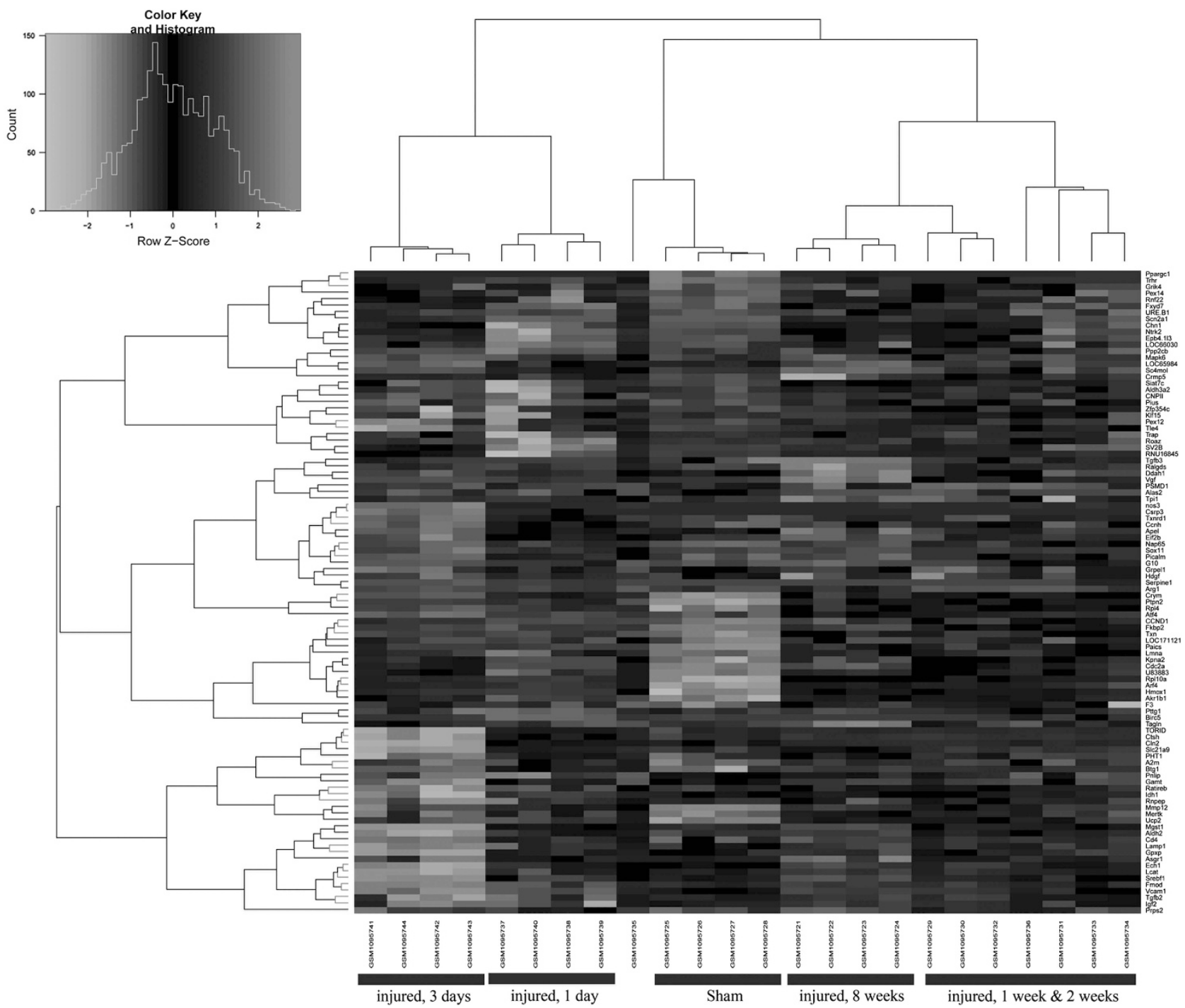

Figure 1 Heatmap for the top 100 temporal genes. A full colour version of this figure is available at the Spinal Cord journal online.

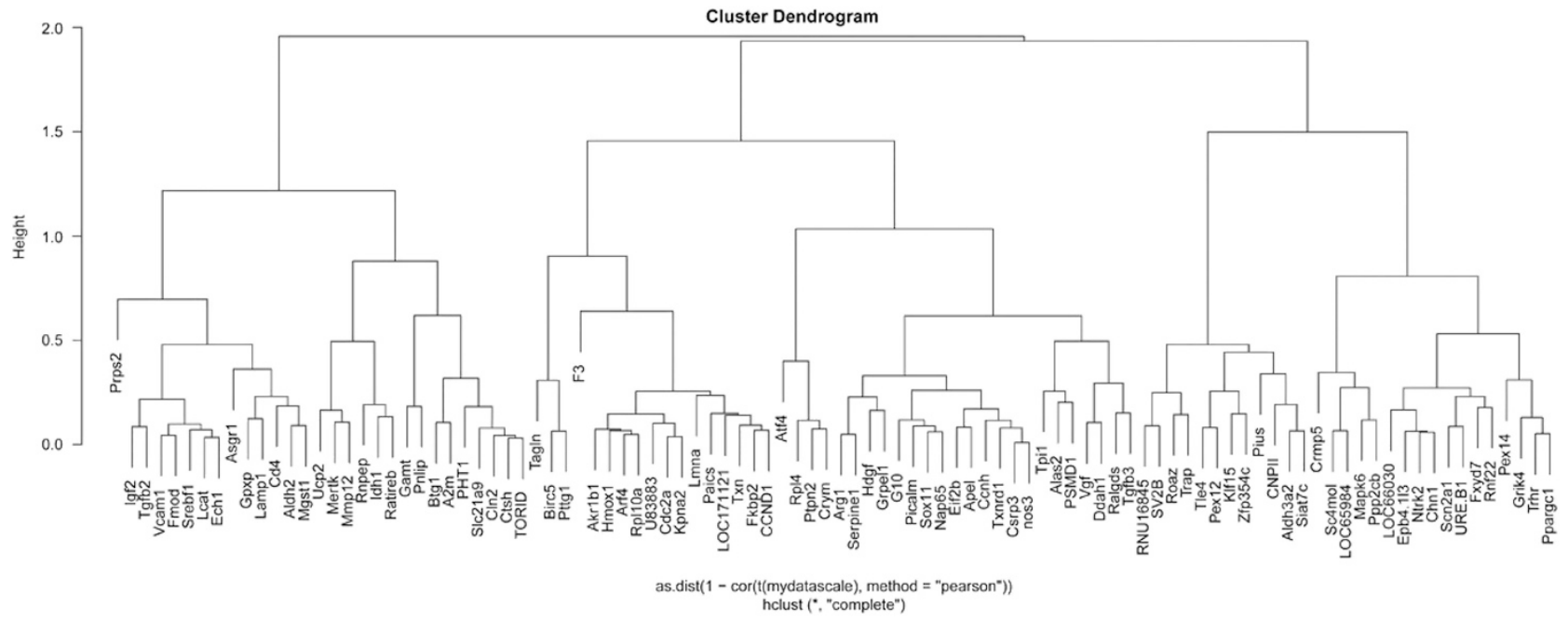

Figure 2 Clustering tree for the top 100 temporal genes. 

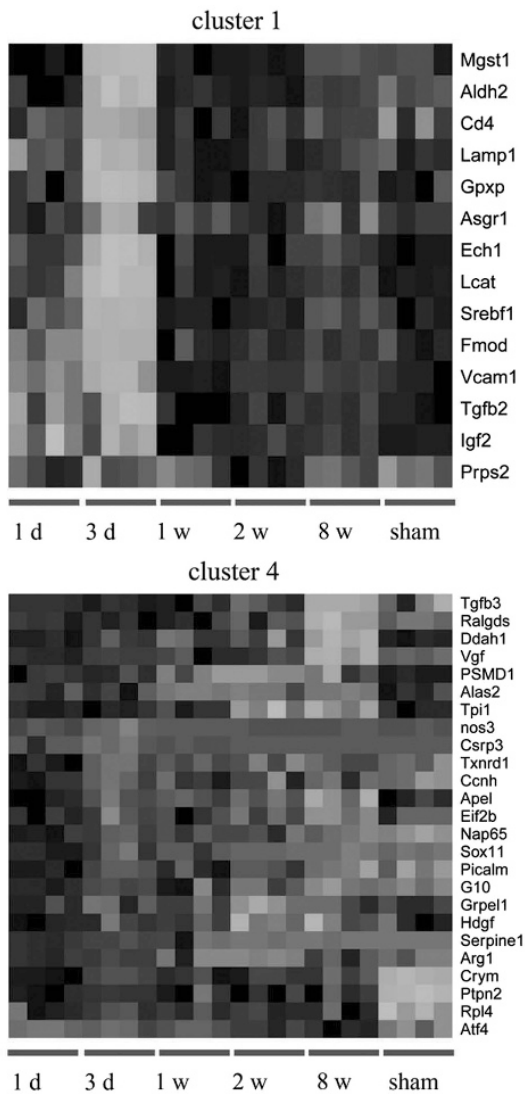

cluster 2

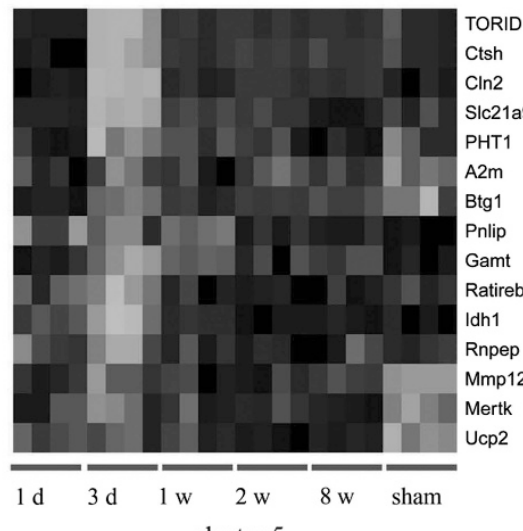

cluster 5

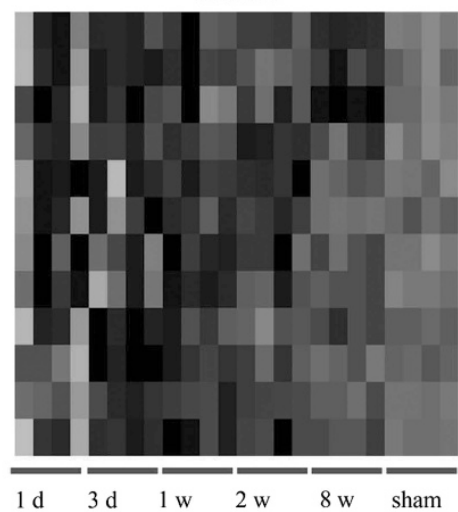

cluster 3



cluster 6



Figure 3 Heatmaps for the top 100 temporal genes in the six clusters (cluster 1, cluster 2, cluster 3, cluster 4, cluster 5 and cluster 6). A full colour version of this figure is available at the Spinal Cord journal online.

Table 1 The top 10 enriched functions for the top 100 temporal genes

\begin{tabular}{|c|c|c|c|c|}
\hline Term & Description & Gene number & Gene symbol & P-value \\
\hline 0051179 & Localisation & 43 & MERTK, CRYM....... & 8.29E-08 \\
\hline 0044699 & Single-organism process & 66 & MERTK, CHN1...... & $2.52 \mathrm{E}-07$ \\
\hline 0010033 & Response to organic substance & 27 & CTSH, FMOD...... & 9.93E-07 \\
\hline 0070887 & Cellular response to chemical stimulus & 26 & CTSH, ALDH2...... & 9.93E-07 \\
\hline 0008152 & Metabolic process & 61 & MERTK, PAICS...... & 9.93E-07 \\
\hline 0044237 & Cellular metabolic process & 58 & CHN1, CRYM...... & 9.93E-07 \\
\hline 0044281 & Small-molecule metabolic process & 30 & PAICS, CHN1...... & $1.08 \mathrm{E}-06$ \\
\hline 0006950 & Response to stress & 33 & MERTK, A2M/...... & $1.08 \mathrm{E}-06$ \\
\hline 0044710 & Single-organism metabolic process & 33 & PAICS,CHN1/ & $1.08 \mathrm{E}-06$ \\
\hline 1901700 & Response to oxygen-containing compound & 19 & CTSH, HMOX1...... & $1.08 \mathrm{E}-06$ \\
\hline
\end{tabular}

Table 2 The enriched pathways for the top 100 temporal genes

\begin{tabular}{llcl}
\hline Terms & Description & Gene number & Gene symbol \\
\hline map00330 & Arginine and proline metabolism & 5 & PAMT, ALDH3A2, NOS3, ALDH2, ARG1 \\
map05210 & Colorectal cancer & 5 & BIRC5,TGFB2, RALGDS, TGFB3,CCND1 \\
map00561 & Glycerolipid metabolism & 4 & ALDH3A2, PNLIP, ALDH2, AKR1B1 \\
map00040 & Pentose and glucuronate interconversions & 3 & ALDH3A2, ALDH2, AKR1B1 \\
map05212 & Pancreatic cancer & 4 & TGFB2, RALGDS, TGFB3,CCND1 \\
map04110 & Cell cycle & 5 & TGFB2,CCNH, PTTG1,TGFB3,CCND1 \\
map04146 & Peroxisome & 4 & IDH1, PEX14, ECH1, PEX12 \\
map00620 & Pyruvate metabolism & 3 & ALDH3A2, ALDH2, AKR1B1 \\
map05144 & Malaria & 3 & VCAM1,TGFB2, TGFB3 \\
map05142 & Chagas disease (American trypanosomiasis) & 4 & TGFB2, TGFB3,SERPINE1,PPP2CB \\
\hline
\end{tabular}


Table 3 The enriched functions and protein domains for genes in the six clusters

\begin{tabular}{|c|c|c|c|c|c|c|}
\hline Cluster & Category & Term & Description & Gene number & Gene symbol & P-value \\
\hline Cluster 2 & GO MF & 0008238 & Exopeptidase activity & 3 & $C T S H, R N P E P, T P P 1$ & 4.20E-02 \\
\hline \multirow[t]{2}{*}{ Cluster 4} & GO BP & 0006527 & Arginine catabolic process & 3 & $A R G 1, D D A H 1, N O S 3$ & 3.77E-03 \\
\hline & GO BP & 0006525 & Arginine metabolic process & 3 & ARG1,DDAH1,NOS3 & $5.65 \mathrm{E}-03$ \\
\hline
\end{tabular}

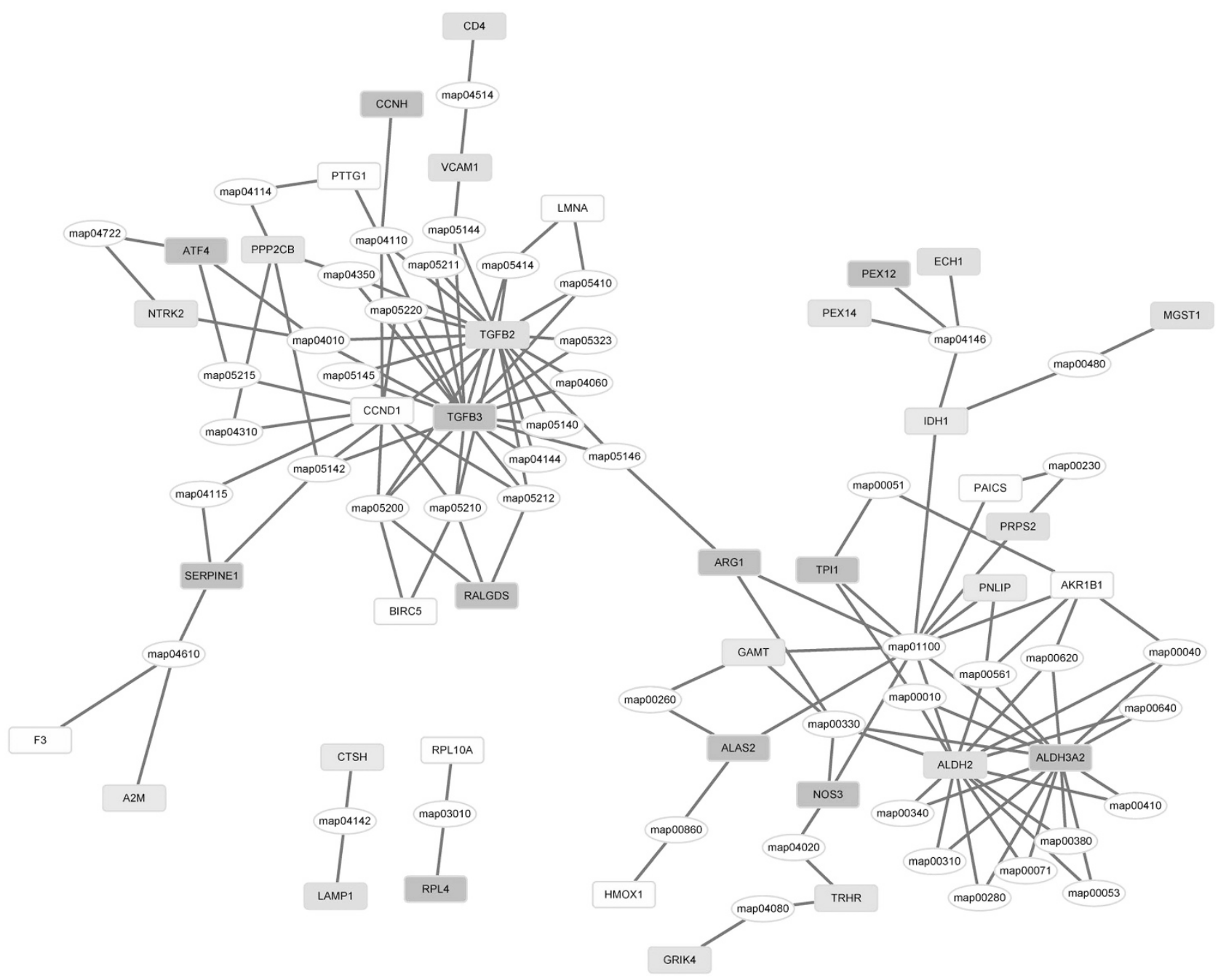

Figure 4 The pathway-gene crosslinking network for genes in the six clusters (cluster 1, cluster 2, cluster 3, cluster 4, cluster 5 and cluster 6). Purple rectangles represent genes in cluster 1 . Pink rectangles indicate genes in cluster 2. Yellow rectangles represent genes in cluster 3 . Green rectangles represent genes in cluster 4. Blue rectangles indicate genes in cluster 5. Orange rectangles represent genes in cluster 6 . Ovals indicate pathways. A full colour version of this figure is available at the Spinal Cord journal online.

arginine and proline metabolism pathways. In addition, $A R G 1$ is the unique connection to the map05146, map01100 and map00330 pathways (Figure 4).

\section{DISCUSSION}

In this study, 1907 temporal genes in the SCI models with a probability $\geqslant 0.95$ were obtained via comparisons with normal samples. Hierarchical clustering showed that the top 100 temporal genes that could be divided into six clusters (cluster 1, cluster 2, cluster 3 , cluster 4 , cluster 5 and cluster 6) exhibited different expression patterns during the time series. Most of the enriched functions for the top 100 temporal genes were in biological process categories. TGF $32, T G F \beta 3, A L D H 2$ and $A L D H 3 A 2$ were correlated with numerous pathways in the pathway-gene crosslinking network. 
The treatment of SCI rats with TGF $\beta$ reduces mononuclear phagocyte numbers around the injury site, reducing their contribution to secondary damage by decreasing lesion volume. ${ }^{32}$ Anti-TGF 1 aggravates secondary injury by inhibiting the anti-inflammatory effect of TGF $\beta 1 .{ }^{33}$ Lagord et $a l .{ }^{34}$ reported the temporal expression and cellular localisation of TGF $\beta$ following SCI, suggesting that TGF $\beta 1$ regulates the inflammatory and neuronal responses, whereas TGF 22 moderates glial/collagen scarring. Thus, TGF $\beta 2$ correlates with the deposition of scar tissue in SCI lesion rather than TGF $\beta 1$ does. In this study, TGF $\beta 2$ and TGF $\beta 3$ expression levels were successively evaluated at 1-3 days and 1-8 weeks following SCI injury. These findings indicate that TGF $\beta 2$ and TGF 33 exhibit a close correlation with the repair of SCI. TGF $\beta 2$ expression levels post SCI were decreased by agmatine treatment, suggesting the therapeutic potential of inhibition of TGF 32 in the treatment of SCI. ${ }^{35}$ As a mitochondrial enzyme metabolises numerous aldehydes, ALDH2 mediates nociception and can be used as a molecular target for controlling pain. ${ }^{36}$ ALDH1A1 protects dopaminergic neurons in substantia nigra pars compacta through inhibiting the formation of cytotoxic $\alpha$-synuclein oligomers and accumulation of dopamine aldehyde intermediates. ${ }^{37}$ Thus, $A L D H 2$ and $A L D H 3 A 2$ might also have important roles in the repair process after SCI.

There are three types of NOS enzymes (constitutive $\mathrm{Ca} 2+$ / calmodulin-dependent neuronal NOS, nNOS; endothelial NOS, eNOS; and inducible calcium-independent isoform, iNOS). These enzymes have a crucial role in the progression of SCI, with degradation of L-arginine to L-citrulline and $\mathrm{NO}^{38}$ Arginase regulates NO levels by competing with NOS for the substrate L-arginine. ${ }^{39}$ Upregulations of eNOS, iNOS and $\mathrm{NAD}(\mathrm{P}) \mathrm{H}$ oxidase cooperate to sustain oxidative and nitrosative stress in the damaged spinal cord tissue. ${ }^{40}$ In addition, the expression of ARG1 may provide a balance for NOS amounts. ${ }^{41}$ Thus, inhibition of arginase may be a promising treatment strategy for neurogenic bladder overactivity in SCI. ${ }^{42}$

Different types of NOS enzymes act in different periods of SCI in rats. In detail, nNOS induces the initial maximal NO increase, and iNOS causes the second wave of NO production. ${ }^{43-45}$ NO induces breakdown of the blood-spinal cord barrier, motor dysfunction and oedema formation in SCI, indicating that inhibitions of $\mathrm{nNOS}$ and $\mathrm{NO}$ production is essential for neuroprotection. ${ }^{38,46}$ Lee et al. ${ }^{47}$ demonstrated that peripheral nerve grafts with acidic fibroblast growth factor treatment markedly induced nNOS protein, and significantly increased eNOS protein compared with injury groups. As previously reported, $T G F \beta$ is associated with $\mathrm{L}$-arginine-dependent NO-mediated mechanisms. ${ }^{48}$ TGF $\beta 2$ and TGF 33 induce Sertoli cell NOS production, suggesting that $T G F \beta 2$ and TGF 3 regulate tight junction-barrier function in Sertoli cells. ${ }^{49}$ In this current study, TGF $\beta 2$ and TGF $\beta 3$ were also associated with arginine catabolic processes through $A R G 1$. These findings might provide a new insight of correlation between TGF $\beta$ and arginine catabolic processes for investigations of SCI mechanism.

Despite the comprehensive analysis, several limitations in the present study should be noted. First, this study was based on the expression profiles data downloaded from the GEO database. Second, the sample size was relatively small, with only four repetitions for each time point. Moreover, all the predictive results were obtained from bioinformatics analysis and must be further confirmed.

In conclusion, we conducted an integrated bioinformatics analysis of genes potentially related to SCI. A total of 1907 temporal genes were identified in the SCI samples. Among the top 100 temporal genes, $T G F \beta 2, T G F \beta 3, A L D H 2, A L D H 3 A 2, A R G 1$ and NOS3 are potentially associated with the mechanisms of SCI. These findings might promote the understanding of the pathogenesis of SCI and lay the foundation for the targeted therapy of SCI. However, further experimental studies are still necessary to unravel their mechanisms of action in SCI.

\section{DATA ARCHIVING}

There were no data to deposit.

\section{CONFLICT OF INTEREST}

The authors declare no conflict of interest.

1 Richards JS, Kewman DG, Pierce CA. Spinal cord injury. Handbook of Rehabilitation Psychology. American Psychological Association: Washington, DC, USA, 2000, pp 11-27.

2 Elliott TR, Rivera P. Spinal cord injury. Handbook of Psychology. Wiley: New York, NY, USA, 2003, pp 415-435.

3 Heinemann AW. Spinal cord injury. Handbook of Health and Rehabilitation Psychology. Springer: New York, NY, USA, 1995, pp 341-360.

4 Plunkett JA, Yu C-G, Easton JM, Bethea JR, Yezierski RP. Effects of interleukin-10 (IL-10) on pain behavior and gene expression following excitotoxic spinal cord injury in the rat. Exp Neurol 2001; 168: 144-154.

5 Zhou Z, Peng X, Insolera R, Fink DJ, Mata M. IL-10 promotes neuronal survival following spinal cord injury. Exp Neurol 2009; 220: 183-190.

6 Zhang L, Ma Z, Smith GM, Wen X, Pressman Y, Wood PM et al. GDNF-enhanced axonal regeneration and myelination following spinal cord injury is mediated by primary effects on neurons. Glia 2009; 57: 1178-1191.

7 Yune TY, Lee JY, Jiang MH, Kim DW, Choi SY, Oh TH. Systemic administration of PEP-1-SOD1 fusion protein improves functional recovery by inhibition of neuronal cell death after spinal cord injury. Free Radic Biol Med 2008; 45: 1190-1200.

8 Kim W, Kim DW, Yoo DY, Chung JY, Hwang IK, Won M-H et al. Neuroprotective effects of PEP-1-Cu, Zn-SOD against ischemic neuronal damage in the rabbit spinal cord. Neurochem Res 2012; 37: 307-313.

9 Okada S, Nakamura M, Katoh H, Miyao T, Shimazaki T, Ishii K et al. Conditional ablation of Stat3 or Socs3 discloses a dual role for reactive astrocytes after spinal cord injury. Nat Med 2006; 12: 829-834.

10 Herrmann JE, Imura T, Song B, Qi J, Ao Y, Nguyen TK et al. STAT3 is a critical regulator of astrogliosis and scar formation after spinal cord injury. J Neurosci 2008; 28: 7231-7243.

11 McKillop WM, Dragan M, Schedl A, Brown A. Conditional Sox9 ablation reduces chondroitin sulfate proteoglycan levels and improves motor function following spinal cord injury. Glia 2013; 61: 164-177.

12 Chamankhah M, Eftekharpour E, Karimi-Abdolrezaee S, Boutros PC, San-Marina S, Fehlings MG. Genome-wide gene expression profiling of stress response in a spinal cord clip compression injury model. BMC Genomics 2013; 14: 1-25.

13 Rivlin A, Tator C. Effect of duration of acute spinal cord compression in a new acute cord injury model in the rat. Surg Neurol 1978; 10: 38-43.

14 Poon PC, Gupta D, Shoichet MS, Tator CH. Clip compression model is useful for thoracic spinal cord injuries: histologic and functional correlates. Spine 2007; 32: 2853-2859.

15 Karimi-Abdolrezaee S, Eftekharpour E, Fehlings MG. Temporal and spatial patterns of Kv1. 1 and Kv1. 2 protein and gene expression in spinal cord white matter after acute and chronic spinal cord injury in rats: implications for axonal pathophysiology after neurotrauma. Eur J Neurosci 2004; 19: 577-589.

16 Nashmi R, Fehlings MG. Mechanisms of axonal dysfunction after spinal cord injury: with an emphasis on the role of voltage-gated potassium channels. Brain Res Rev 2001; 38: 165-191.

17 Yong L, Liu S, Zhang S, Deng Fa. Smoothing Newton method for absolute value equations based on aggregate function. Int J Phys Sci 2011; 6: 5399-5405.

18 Hastie T, Tibshirani R, Narasimhan B, Chu G, Hastie T, Tibshirani R et al. Impute: imputation for microarray data. Bioinformatics 2001; 17: 520-525.

19 Bolstad B. preprocessCore: A Collection of Pre-Processing Functions. R Package Version 1.02013.

20 Gentleman R, Carey V, Huber W, Hahne F. Genefilter: Methods for Filtering Genes from Microarray Experiments. R Package Version 1.0 2011.

21 Aryee MJ, Gutiérrez-Pabello JA, Kramnik I, Maiti T, Quackenbush J. An improved empirical Bayes approach to estimating differential gene expression in microarray time-course data: BETR (Bayesian Estimation of Temporal Regulation). BMC Bioinformatics 2009; 10: 409.

22 Díaz-Uriarte R, De Andres SA. Gene selection and classification of microarray data using random forest. BMC Bioinformatics 2006; 7: 1.

23 Warnes GR, Bolker B, Bonebakker L, Gentleman R, Huber W, Liaw A et al. gplots: various R programming tools for plotting data. R Package Version 2.42009.

24 Wang J. Pearson Correlation Coefficient. Encyclopedia of Systems Biology. Springer, New York, NY, USA, 2013, pp 1671.

25 Smith B, Williams J, Schulzekremer S. The ontology of the gene ontology. AMIA Annu Symp Proc 2003; 6: 609-613.

26 Altermann E, Klaenhammer TR. PathwayVoyager: pathway mapping using the Kyoto Encyclopedia of Genes and Genomes (KEGG) database. BMC Genomics 2005; 6: 60.

27 Yu G, Wang L-G, Han Y, He Q-Y. clusterProfiler: an R package for comparing biological themes among gene clusters. OMICS 2012; 16: 284-287. 
28 Thissen D, Steinberg L, Kuang D. Quick and easy implementation of the Benjamini-Hochberg procedure for controlling the false positive rate in multiple comparisons. J Educ Behav Stat 2002; 27: 77-83.

29 Chen Y-A, Tripathi LP, Mizuguchi K. TargetMine, an integrated data warehouse for candidate gene prioritisation and target discovery. PLOS ONE 2011; 6: e17844.

30 Holm S. A simple sequentially rejective multiple test procedure. Scand Stat Theory Appl 1979; 6: 65-70

31 Shannon P, Markiel A, Ozier O, Baliga NS, Wang JT, Ramage D et al. Cytoscape: a software environment for integrated models of biomolecular interaction networks. Genome Res 2003; 13: 2498-2504.

32 Tyor WR, Avgeropoulos N, Ohlandt G, Hogan EL. Treatment of spinal cord impact injury in the rat with transforming growth factor- $\beta$. J Neurol Sci 2002; 200: 33-41.

33 King V, Phillips J, Brown R, Priestley J. The effects of treatment with antibodies to transforming growth factor $\beta 1$ and $\beta 2$ following spinal cord damage in the adult rat. Neuroscience 2004; 126: 173-183.

34 Lagord C, Berry M, Logan A. Expression of TGF $\beta 2$ but not TGF $\beta 1$ correlates with the deposition of scar tissue in the lesioned spinal cord. Mol Cell Neurosci 2002; 20 69-92.

35 Kim JH, Lee YW, Park YM, Park KA, Park SH, Lee WT et al. Agmatine-reduced collagen scar area accompanied with surface righting reflex recovery after complete transection spinal cord injury. Spine 2011; 36: 2130-2138.

36 Zambelli VO, Gross ER, Chen C-H, Gutierrez VP, Cury Y, Mochly-Rosen D. Aldehyde dehydrogenase-2 regulates nociception in rodent models of acute inflammatory pain. Sci Transl Med 2014; 6: 251ra118.

37 Liu G, Yu J, Ding J, Xie C, Sun L, Rudenko I et al. Aldehyde dehydrogenase 1 defines and protects a nigrostriatal dopaminergic neuron subpopulation. J Clin Invest 2014, 124: 3032-3046.

38 Diaz-Ruiz A, Vergara P, Perez-Severiano F, Segovia J, Guizar-Sahagún G, Ibarra A et al. Cyclosporin-A inhibits constitutive nitric oxide synthase activity and neuronal and endothelial nitric oxide synthase expressions after spinal cord injury in rats. Neurochem Res 2005; 30: 245-251.
39 Luiking YC, Engelen MP, Deutz NE. Regulation of nitric oxide production in health and disease. Curr Opin Clin Nutr Metab Care 2010; 13: 97.

40 Vaziri ND, Lee Y-S, Lin C-Y, Lin VW, Sindhu RK. NAD (P) H oxidase, superoxide dismutase, catalase, glutathione peroxidase and nitric oxide synthase expression in subacute spinal cord injury. Brain Res 2004; 995: 76-83.

41 Neill MA, Aschner J, Barr F, Summar ML. Quantitative RT-PCR comparison of the urea and nitric oxide cycle gene transcripts in adult human tissues. Mol Genet Metab 2009, 97: 121-127.

42 Sasatomi K, Hiragata S, Miyazato M, Chancellor MB, Morris SM Jr, Yoshimura N. Nitric oxide-mediated suppression of detrusor overactivity by arginase inhibitor in rats with chronic spinal cord injury. Urology 2008; 72: 696-700.

43 Nakahara S, Yone K, Setoguchi T, Yamaura I, Arishima Y, Yoshino S et al. Changes in nitric oxide and expression of nitric oxide synthase in spinal cord after acute traumatic injury in rats. J Neurotrauma 2002; 19: 1467-1474.

44 Díaz-Ruiz A, Ibarra A, Pérez-Severiano F, Guízar-Sahagún G, Grijalva I, Ríos C. Constitutive and inducible nitric oxide synthase activities after spinal cord contusion in rats. Neurosci Lett 2002; 319: 129-132.

45 Chatzipanteli K, Garcia R, Marcillo AE, Loor KE, Kraydieh S, Dietrich WD. Temporal and segmental distribution of constitutive and inducible nitric oxide synthases after traumatic spinal cord injury: effect of aminoguanidine treatment. J Neurotrauma 2002; 19: 639-651.

46 Sharma HS, Badgaiyan RD, Alm P, Mohanty S, Wiklund L. Neuroprotective effects of nitric oxide synthase inhibitors in spinal cord injury-induced pathophysiology and motor functions. Ann NY Acad Sci 2005; 1053: 422-434.

47 Lee Y-S, Sindhu RK, Lin C-Y, Ehdaie A, Lin VW, Vaziri ND. Effects of nerve graft on nitric oxide synthase, NAD (P) $\mathrm{H}$ oxidase, and antioxidant enzymes in chronic spinal cord injury. Free Radic Biol Med 2004; 36: 330-339.

48 Gazzinelli R, Oswald J, Hienys S, James S, Sher A. The microbicidal activity of IFNc treated macrophages against $T$. cruzi involves an L-arginine-dependent nitrogen oxide-mediated mechanism inhibitable by IL-10 and TGF-b. Eur J Immunol 1992; 22: 2501-2506.

49 Lee NP, Cheng CY. Nitric oxide/nitric oxide synthase, spermatogenesis, and tight junction dynamics. Biol Reprod 2004; 70: 267-276. 